\title{
Allergic Contact Dermatitis Syndrome Due to Tocopherol Acetate, in Addition to Glycyrrhetinic Acid
}

\author{
Kentaro Ohko, Akiko Ito, Masaaki Ito \\ Division of Dermatology, Graduate School of Medical and Dental Sciences, Niigata University, Niigata, Japan \\ Email: kentar05@med.niigata-u.ac.jp
}

Received December $18^{\text {th }}, 2011$; revised December $30^{\text {th }}, 2011$; accepted January $13^{\text {th }}, 2012$

\begin{abstract}
Natural vitamin $\mathrm{E}$ is suggested to have an antioxidant function. However, the synthetic form of vitamin E, $D L$-tocopherol, which has been widely used in topical ointments, may cause allergic contact dermatitis. Here, we report a case of allergic contact dermatitis with erythema multiforme-like eruption caused by a topical ointment. Patch testing indicated a positive allergic reaction to an anti-inflammatory ointment the patient had been using and its ingredient, $D L$ alpha-tocopheryl acetate (vitamin E). In addition, a positive reaction to glycyrrhetinic acid was observed. Both vitamin E and glycyrrhetinic acid are useful ingredients of topical applications. However, the possibility that both can cause contact dermatitis, albeit rarely, should be considered.
\end{abstract}

Keywords: Allergic Contact Dermatitis; Tocopheryl Acetate; Vitamin E; Enoxolone; 18-Glycyrrhetinic Acid

\section{Case Report}

A 39-years-old woman without any notable medical history had been using a skin lotion on her face, neck and arms for about 7 months without any problems. However, itchy erythema developed on the bilateral cubital fossae, and for this the patient began to apply an anti-pruritic and anti-inflammatory ointment that is widely used in Japan. Two weeks later, the rash had spread to her neck and upper extremities, and she stopped applying her skin lotion, while continuing with the ointment. She visited a local dermatologist and was prescribed topical corticosteroids (deprodone propionate for the trunk and extremities, and alclometasone dipropionate for the face). After two days, an erythema multiforme-like eruption and bullae spread to both extremities, pustules developed on the neck, and the patient became febrile. Therefore she visited our hospital and was admitted (Figure 1). Her con- dition was finally improved after administration of a short course of oral corticosteroid (prednisolone $30 \mathrm{mg} /$ day for three days, followed by gradual tapering). Patch testing demonstrated a positive allergic reaction to the anti-inflammatory ointment itself, its ingredients including $D L$-alpha-tocopheryl acetate $5 \%$ pet, glycyrrhetinic acid $2 \%$ pet, and glycyrrhetinic acid $0.2 \%$, and the skin lotion (containing $0.002 \% D$-gamma-tocopherol) based on the criteria of the International Contact Dermatitis Research Group. However, there was no reaction to $D L$ alpha-tocopheryl acetate $0.5 \%$ pet (Table 1). Control patch tests were performed on 5 healthy volunteers using $D L$-alpha-tocopheryl acetate and glycyrrhetinic acid, but no allergic or irritant reaction was elicited.

\section{Discussion}

Both vitamin E and glycyrrhetinic acid are widely used

Table 1. Patch test results.

\begin{tabular}{lcccc}
\hline & & day 2 & day 3 & day 7 \\
\hline Ointment (she used) & as is & ++ & ++ & ++ \\
glycyrrhetinic acid & $0.2 \%$ pet & + & ++ & ++ \\
glycyrrhetinic acid & $2.0 \%$ pet & + & - & ++ \\
$D L-\alpha$-tocopheryl acetate & $0.5 \%$ pet & - & ++ & - \\
$D L-\alpha$-tocopheryl acetate & $5.0 \%$ pet & ++ & ++ \\
skin lotion (containing $0.002 \% D-\gamma$-tocopherol) & as is & - & ++ & ++ \\
petroleum jelly & & - & - & ++ \\
\hline
\end{tabular}

Readings were performed according to the International Contact Dermatitis Research Group criteria on day 2, day 3 and day 7 ; + is weak (nonvesicular) positive reaction; erythema, infiltration, possibly papules; ++ is strong (vesicular) positive reaction; erythema, infiltration, papules, vesicles. 


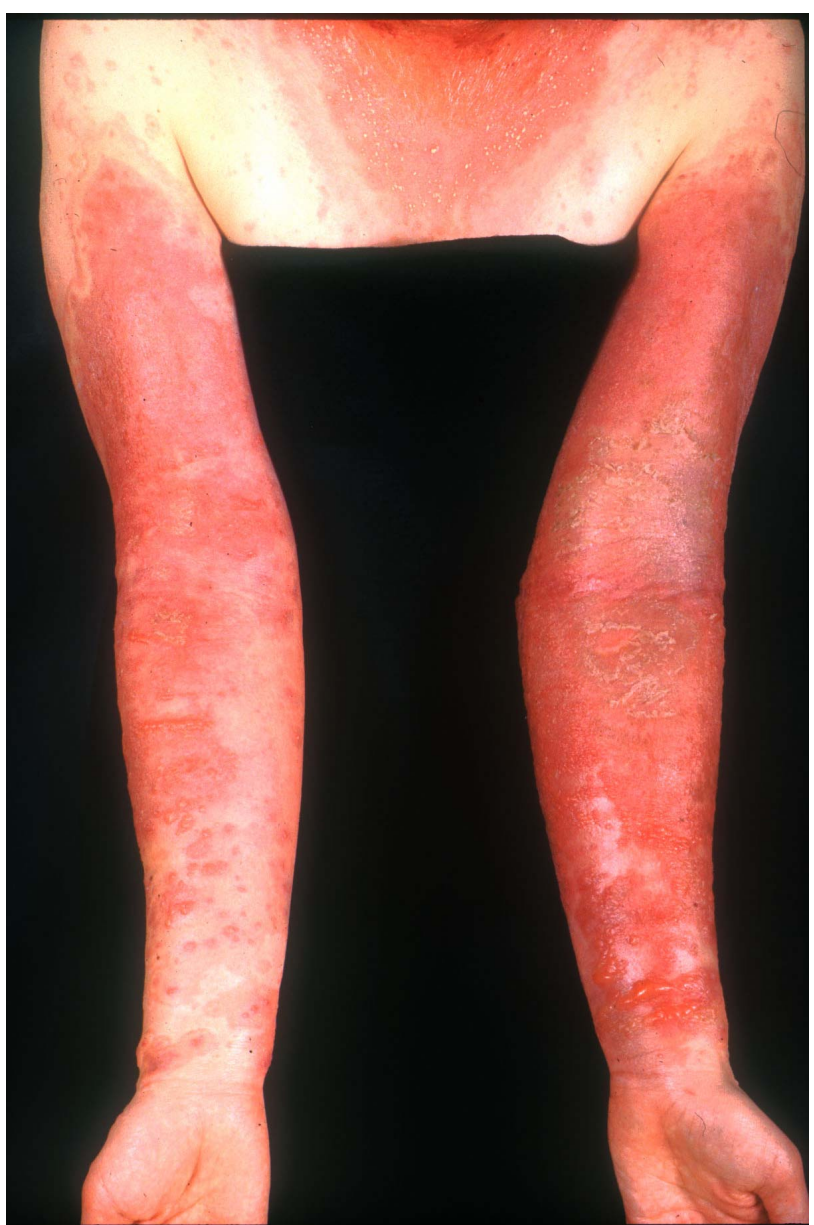

Figure 1. Erythema and bullae on both arms.

in topical ointments. Natural vitamin E is suggested to have an antioxidant function. Several forms of vitamin $\mathrm{E}$ exist, and non-esterified $D$-alpha-tocopherol has the greatest biologic activity. The synthetic form, $D L$-tocopherol is a mixture of eight stereoisomers, and the esterified forms (usually the acetate ester) are more stable, although their antioxidant potential is minimal [1]. Furthermore, the $D L$-form of vitamin $\mathrm{E}$ has been reported to cause allergic contact dermatitis when applied topically [2-9], and only tocopherol causes an erythema-multiforme-like eruption [4]. Our patient showed no allergic reaction to $D L$-alpha-tocopheryl acetate $0.5 \%$, which was an ingredient of the cream she was using. This was compatible with the fact that a $10 \%$ pet patch-test concentration of tocopheryl acetate has been recommended [10]. However, Matsumura [8] has reported a case of allergy elicited by much lower concentrations $(0.5 \%, 0.25 \%)$. Enoxolone (18-glycyrrhetinic acid), on the other hand, a metabolite of glycyrrhizinic acid, is a widely used ingredient of topical anti-inflammatory preparations [11], and some cases of sensitization to it have been reported [11-13].

We suspect that our patient had become sensitized to $D L$-alpha-tocopheryl acetate and glycyrrhetinic acid in-<smiles>CC(=O)Oc1c(C)c(C)c2c(c1C)CCC(C)(CCCC(C)CCCC(C)CCCC(C)C)O2</smiles>

$D L$ - $\alpha$-tocopheryl acetate (CAS 7695-91-2)<smiles>Cc1c(O)cc2c(c1C)O[C@](C)(CCCC(C)CCCC(C)CCCC(C)C)CC2</smiles>

$D$ - $\gamma$-tocopherol (CAS 54-28-4)

Figure 2. Chemical structure of all-rac-alpha-tocopheryl acetate $(D L$-alpha-tocopheryl acetate) and $D$-gamma-tocopherol.

dependently, as their chemical structures are quite different. On the other hand, the chemical structures of the two tocopherol forms are quite similar (Figure 2). Although vitamin E and glycyrrhetinic acid are widely used in ointments and cosmetics, and are useful ingredients, the present case suggests that both can cause contact dermatitis, albeit rarely.

\section{REFERENCES}

[1] K. E. Burke, "Interaction of Vitamins C and E as Better Cosmeceuticals," Dermatologic Therapy, Vol. 20, No. 5, 2007, pp. 314-321.

doi:10.1111/j.1529-8019.2007.00145.x

[2] R. H. Brodkin and J. Bleiberg, "Sensitivity to Topically Applied Vitamin E," Archives of Dermatology, Vol. 92, No. 1, 1965, pp. 76-77.

doi:10.1001/archderm.1965.01600130082016

[3] J. Roed-Petersen and N. Hjorth, "Patch Test Sensitization from $D L$-alpha-tocopherol (Vitamin E)," Contact Dermatitis, Vol. 1, No. 6, 1975, p. 391.

[4] H. Saperstein, M. Rapaport and R. L. Rietschel, "Topical Vitamin E as a Cause of Erythema Multiforme-Like Eruption," Archives of Dermatology, Vol. 120, No. 7, 1984, pp. 906-908. doi:10.1001/archderm.1984.01650430092016

[5] A. C. de Groot, P. J. Berretty, C. J. van Ginkel, C. W. den Hengst, J. van Ulsen and J. W. Weyland, "Allergic Contact Dermatitis from Tocopheryl Acetate in Cosmetic Creams," Contact Dermatitis, Vol. 25, No. 5, 1991, pp. 302-304. doi:10.1111/j.1600-0536.1991.tb01878.x

[6] B. Garcia-Bravo and P. Mozo, "Generalized Contact Dermatitis from Vitamin E," Contact Dermatitis, Vol. 26, No. 4, 1992, p. 280.

doi:10.1111/j.1600-0536.1992.tb00258.x

[7] D. Manzano, A. Aguirre, J. Gardeazabal, X. Eizaguirre 
and J. L. Diaz Perez, "Allergic Contact Dermatitis from Tocopheryl Acetate (Vitamin E) and Retinol Palmitate (Vitamin A) in a Moisturizing Cream," Contact Dermatitis, Vol. 31, No. 5, 1994, p. 324.

doi:/10.1111/j.1600-0536.1994.tb02030.x

[8] T. Matsumura, T. Nakada and M. Iijima, "Widespread Contact Dermatitis from Tocopherol Acetate," Contact Dermatitis, Vol. 51, No. 4, 2004, pp. 211-212. doi:10.1111/j.0105-1873.2004.0424b.x

[9] A. Ramirez Santos, V. Fernandez-Redondo, L. Perez Perez, J. Concheiro Cao and J. Toribio, "Contact Allergy from Vitamins in Cosmetic Products," Dermatitis, Vol. 19, No. 3, 2008, pp. 154-156.

[10] R. Rietshel and J. Fowler, "Fisher's Contact Dermatitis 6,” BC Decker Inc., Hamilton, 2008.
[11] S. Tanaka, T. Otsuki, Y. Matsumoto, R. Hayakawa and M. Sugiura, "Allergic Contact Dermatitis from Enoxolone," Contact Dermatitis, Vol. 44, No. 3, 2001, p. 192. doi:10.1034/j.1600-0536.2001.440308-13.x

[12] J. C. Fernandez, P. Gamboa, I. Jauregui, G. Gonzalez and I. Antepara, "Concomitant Sensitization to Enoxolone and Mafenide in a Topical Medicament," Contact Dermatitis, Vol. 27, No. 4, 1992, p. 262. doi:10.1111/j.1600-0536.1992.tb03263.x

[13] N. Oiso, T. Ota, E. Yoshinaga, H. Endo, S. Kawara and A. Kawada, "Allergic Contact Dermatitis Mimicking Atopic Dermatitis Due to Enoxolone in a Topical Medicament," Contact Dermatitis, Vol. 54, No. 6, 2006, p. 351. doi:10.1111/j.0105-1873.2006.0645i.x 